\title{
Depuración aerobia de los efluentes resultantes del proceso de biometanización del alpechín.
}

\author{
Por R. Borja Padilla, Mª. M. Durán Barrantes y M. Luque González. \\ Instituto de la Grasa y sus Derivados (C.S.I.C.). Avda. Padre \\ García Tejero 4. 41012-SEVILLA.
}

RESUMEN

Depuración aerobia de los efluentes resultantes del proceso de biometanización del alpechin.

Se ha efectuado un estudio del proceso de depuración aerobia, en régimen discontinuo, de los efluentes procedentes del proceso de depuración anaerobia o biometanización del alpechín.

Se comprueba que el $83 \%$ de la materia orgánica presente en este efluente se elimina a partir del tercer dia de fermentación.

La eliminación de sustrato sigue una cinética de orden cero para altas concentraciones del mismo y una cinética de primer orden para bajas concentraciones de materia orgánica, es decir, durante los últimos dias de fermentación.

Se aplica el modelo de Michaelis-Menten de eliminación de sustrato para la obtención de los parámetros cinéticos $q_{\max }$ y $\mathrm{K}_{\mathrm{s}}$ que rigen este proceso.

PALABRAS-CLAVE: Alpechín - Cinética - Concentración de sustrato - Depuración aerobia - Efluentes del proceso de biometanización.

\section{SUMMARY}

Aerobic purification of the effluents produced in the olive mill wastewater biomethanation process.

A study of aerobic treatment in batch regime of the effluents produced in the olive mill wastewater biomethanation process was carried out.

An $83 \%$ of effluents organic substances was removal after the third day of fermentation.

The substrate removal rate follows a zero-order kinetic for high concentrations, and a first-order kinetic for low organic matter concentration, during the last days of fermentation.

The kinetic parameters $\left(q_{\max }\right.$ and $\left.\mathrm{K}_{\mathrm{g}}\right)$ were obtained from MichaelisMenten model.

KEY-WORDS: Aerobic purification - Effluents of the biomethanation process - Kinetics - Olive mill wastewater - Substrate concentration.

\section{INTRODUCCION}

Entre las posibles soluciones y tecnologías disponibles de cara a la depuración y aprovechamiento del alpechín, destaca la digestión anaerobia o biometanización, cuyas ventajas más notables son las siguientes (1) (2):

- Las necesidades energéticas son muy pequeñas.

- Las bacterias anaerobias transforman la sustancia orgánica en gas metano.

- Los lodos formados sólo suponen un $10 \%$ de la demanda química de oxígeno (D.Q.O.).

- Escaso requerimiento de nutrientes.

La aplicación de este proceso disminuye en un 80$90 \%$ la materia orgánica de este agua residual, quedando un efluente con un cierto contenido en demanda química de oxígeno (4-5 g/l) que es necesario eliminar antes de su vertido o posible reutilización.

De entre las alternativas disponibles para la eliminación de esta fracción de DQO residual, no biodegradada en el proceso anaerobio, pueden aplicarse los procesos biológicos aerobios que en el caso de estos efluentes suelen ser bastante rápidos dado el alto nivel de degradación existente en el sustrato por la acción inicial de la digestión anaerobia (3).

El objetivo de este trabajo es el estudio del proceso de depuración aerobia de los efluentes resultantes del proceso de biometanización del alpechín, con obtención de las constantes cinéticas que rigen el mismo.

\section{PARTE EXPERIMENTAL}

\subsection{Equipo experimental}

Para llevar a cabo el proceso de depuración se utiliza un reactor de 2 litros de capacidad, construido en metacrilato, que contiene volumen total de líquido de 1,55 litros. El grado de mezcla adecuado se consigue con una pequeña turbina que gira a $360 \mathrm{rpm}$. Se utiliza un flujo de aire de $750 \mathrm{ml} / \mathrm{minuto}$ suministrado a través de un difusor cerámico. 
El reactor se encuentra en el interior de una cámara termostatizada a $25^{\circ} \mathrm{C}$.

\subsection{Características de los efluentes del proceso} de biometanización del alpechín.

Las características de los efluentes del proceso de depuración anaerobia o biometanización del alpechín utilizados se muestran en la Tabla I.
Estos efluentes objeto de estudio proceden del proceso de depuración anaerobia de un alpechín de sistema continuo (almazara experimental del Instituto de la Grasa), cuya DQO inicial es de $45 \mathrm{~g} / \mathrm{l}$.

2.3. Acondicionamiento del agua residual objeto de estudio.

Teniendo en cuenta que los nutrientes, principalmente nitrógeno y fósforo, son en algunas ocasiones el factor

Tabla I

Características de los efluentes del proceso de biometanización del alpechín utilizados.

\begin{tabular}{|l|c|}
\hline$p H$ & 7,2 \\
\hline$D Q O$ & $4,8 \mathrm{~g} / 1$ \\
\hline $\begin{array}{l}\text { Acidez volátil } \\
\text { (acido acetico) }\end{array}$ & $150 \mathrm{mg} / \mathrm{I}$ \\
\hline Alcalinidad (CaCo ) & $450 \mathrm{mg} / 1$ \\
\hline Mitrogeno amoniacal & $20 \mathrm{mg} / 1$ \\
\hline
\end{tabular}

limitante del crecimiento y síntesis celular y dado el escaso contenido de los mismos en este agua residual (20 y $12 \mathrm{mg} / \mathrm{l}$ respectivamente), se adicionan ambos elementos para mantener la relación DQO:N:P en 100:5:1, valores recomendables para el crecimiento adecuado de las bacterias responsables del proceso y la subsiguiente degradación de la materia residual (4) (5).

\subsection{Aclimatación de la biomasa aerobia.}

Previamente a la realización de los experimentos y para la aclimatación de la biomasa aerobia del proceso se llevan a cabo unos experimentos iniciales de adaptación, consistentes en la siembra inicial con $100 \mathrm{ml}$ de lodos procedentes de una depuradora aerobia convencional (Empresa Municipal de Aguas de Sevilla S.A., EMASESA) en el reactor que contiene 1,5 litros del agua residual objeto de estudio; éste se mantiene en agitación y aireación durante un período de 7 días. Al cabo de este tiempo y tras una breve decantación se separa el líquido sobrenadante de los lodos bacterianos que permanecen en el fondo del reactor, añadiéndose nuevamente el mismo volumen de agua residual. Se repite esta operación durante 5 semanas consecutivas, de tal manera que durante este período de tiempo los niveles de eliminación de la DQO del agua se mantienen constantes, lográndose condiciones de equilibrio estables en el efluente tratado. Conseguido este objetivo se separa la biomasa desarrollada y adaptada por decantación y se inician los experimentos.

\subsection{Procedimiento experimental.}

Para la ejecución de los experimentos se toman 100 $\mathrm{ml}$ de esta biomasa adaptada (cuyo contenido en sólidos en suspensión volátiles es de $4,5 \mathrm{~g} / \mathrm{l})$ y se completa hasta los 1,5 litros de volumen con el agua residual a tratar. Se mantienen en agitación homogénea y aireación a la temperatura constante de $25^{\circ} \mathrm{C}$ durante un período de 7 días, en régimen discontinuo.

Diariamente se toma una muestra de $20 \mathrm{ml}$ del interior del reactor para determinar el contenido en sólidos en suspensión volátiles (SSV) y el valor de la DQO del agua con el transcurso del tiempo.

\subsection{Técnicas analíticas.}

Los análisis de los parámetros de caracterización del agua se realizan de acuerdo con el "Standard Methods for the Examination of Water and Wastewater» (6).

\section{RESULTADOS EXPERIMENTALES}

En la Figura 1 se recoge la variación de la concentración de sustrato del agua residual estudiada, medida como DQO $(\mathrm{g} / \mathrm{l})$, en función del tiempo de fermentación (días).

En la Tabla II se exponen los valores de concentración de sólidos en suspensión volátiles ( $\mathrm{g} / \mathrm{l}$ ) para los distintos tiempos a lo largo de este proceso de depuración aerobia.

Todos los experimentos se realizan por duplicado.

\section{DISCUSION DE LOS RESULTADOS}

Como se observa en la Figura 1 , más del $83 \%$ de la materia orgánica inicial (DQO) se elimina a partir del tercer día de aireación, existiendo una disminución muy 


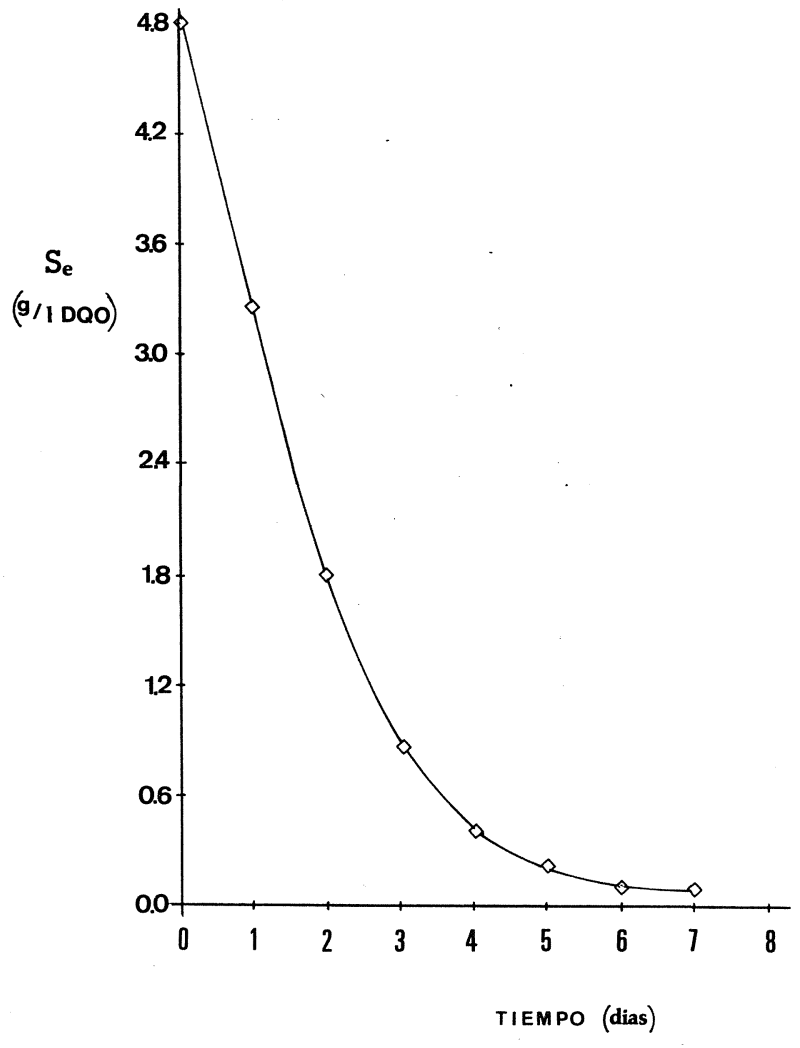

Figura 1

Variación de la concentración de sustrato, evaluada como DQO, en función del tiempo de fermentación. acusada de este parámetro, hasta alcanzar un valor mínimo de $0,1 \mathrm{~g} / \mathrm{l}$, cantidad correspondiente a la fracción no biodegradable. Este valor se mantiene constante a partir del 60 día de aireación.

Como se aprecia en la Tabla II, durante los cinco primeros días de fermentación existe un aumento gradual del contenido de biomasa bacteriana (SSV), período correspondiente a la fase de síntesis celular, al existir en el medio cantidad de sustrato suficiente para el crecimiento y desarrollo de nuevos microorganismos. A partir del quinto día de aireación, existe un paulatino descenso en el contenido de lodos bacterianos, debido a que la velocidad de destrucción celular supera a la de síntesis; este tramo corresponde a la fase de metabolismo endógeno del sistema.

Se pueden distinguir dos valores puntuales de tiempo de digestión, equiparables a otros tantos tiempos de residencia típicos del reactor que opera en régimen contínuo. En primer término, como se observa en la Figura 1, durante el tercer día de fermentación el valor de la DQO se encuentra en $0,8 \mathrm{~g} / \mathrm{l}$, correspondiente a una eliminación del $83,3 \%$ respecto del valor inicial $(4,8 \mathrm{~g} / \mathrm{l})$ mientras que el contenido en lodos bacterianos está en $1,95 \mathrm{~g} / \mathrm{l}$ de SSV (Tabla II). Estos valores se encuentran dentro del intervalo habitual correspondiente al proceso de «lodos activos" convencional del reactor continuo (80-90\% de reducción de DQO y 1,5-3,0 g/l de SSV) (7).

En segundo lugar, durante el quinto día de digestión se alcanza el valor máximo en la concentración de SSV (3,45 g/l, Tabla II), lográndose una eliminación del $98,5 \%$ de la DQO de este residuo. Estos valores se encuentran

Tabla II

Valores de la concentración de sólidos en suspensión volátiles $(\mathrm{g} / \mathrm{l})$ en función del tiempo de digesstión (dias).

\begin{tabular}{|c|c|}
\hline Tiempo (dias) & ssv $(g / 1)$ \\
\hline 0 & 0,30 \\
\hline 1 & 0,60 \\
\hline 2 & 1,25 \\
\hline 3 & 1,95 \\
\hline 4 & 2,65 \\
\hline 5 & 3,45 \\
\hline 6 & 3,05 \\
\hline 7 & 2,80 \\
\hline
\end{tabular}

dentro del margen correspondiente a procesos de «aireación extensiva" para el reactor en régimen continuo (90-100\% de eliminación de DQO y de 3,0 a $5,0 \mathrm{~g} / \mathrm{l}$ de SSV) (7).

A partir de la representación correspondiente a la variación de concentración de sustrato con el tiempo (Figura 1), se obtienen los valores puntuales de la velocidad de eliminación de sustrato, por medio de la pendiente de la tangente a la curva para los distintos tiempos de digestión. En la Tabla III se exponen los valores de este parámetro para cada concentración de sustrato y tiempo de fermentación. Del estudio detallado de esta Tabla se observan dos hechos destacables:

a) Para altas concentraciones de sustrato $\left(S_{\theta} \geq 1,8 \mathrm{~g} /\right.$ I de DQO), es decir durante los dos primeros días de fermentación, la velocidad de eliminación de sustrato se 
mantiene constante $\left(\mathrm{dS}_{\mathrm{e}} / \mathrm{dt}=1,52\right)$ e independiente de la concentración del mismo; por tanto para este intervalo de concentraciones el proceso de eliminación de sustrato sigue una cinética de orden cero.

b) Para bajas concentraciones de sustrato $\left(\mathrm{Se}_{0} \leq 0,8 \mathrm{~g} /\right.$ I de DQO), correspondientes a tiempos de digestión entre 3 y 6 días, la velocidad de eliminación de sustrato es directamente proporcional a la concentración del mismo. Esto nos indica que para este rango dé concentraciones, la velocidad de eliminación presenta una cinética de primer orden. De esta forma, utilizando el método de mínimos cuadrados, los valores de velocidad de eliminación se pueden linealizar frente a los valores de concentraciones de sustrato, $S_{e}$, para los días $3,4,5$ y 6 , obteniéndose los siguientes parámetros con sus límites de confianza:

Ordenada en el origen $=0,009 \pm 0,001$

Pendiente $=0,68 \pm 0,03$

(Coeficiente de regresión lineal $=0,999$ )
Se observa que la ordenada en el origen de esta correlación es prácticamente nula y existe un buen ajuste lineal entre estos parámetros para este rango de bajas concentraciones de sustrato, lo que demuestra que la cinética de eliminación de materia orgánica es de primer orden en este intervalo de concentraciones.

\section{Determinación de los parámetros cinéticos del proceso.}

Para obtener los datos cinéticos que rigen este proceso de depuración aerobia se aplica la ecuación de Michaelis-Menten de utilización de sustrato (7):

$$
q=q_{\max } \cdot \frac{S_{\theta}}{\left(K_{s}+S_{\theta}\right)}
$$

Tabla III

Valores de la velocidad de eliminación de sustrato, $\mathrm{dS}_{\mathrm{e}} / \mathrm{dt}$, para los distintos tiempos $y$ concentraciones de sustrato, $S_{e^{\prime}}$ del proceso de digestión.

\begin{tabular}{|c|c|c|}
\hline Tiempo (dias) & $s_{\rho}(g / 1)$ & $d S_{\rho} / d t\left(g \cdot 1^{-1} d^{2} a^{-1}\right)$ \\
\hline 1 & 3,3 & 1,52 \\
\hline 2 & 1,8 & 1,52 \\
\hline 3 & 0,8 & 0,54 \\
\hline 4 & 0,4 & 0,27 \\
\hline 5 & 0,2 & 0,13 \\
\hline 6 & 0,1 & 0,07 \\
\hline
\end{tabular}

Donde: $q$ = Velocidad específica de eliminación de sustrato, definida como la variación de la concentración de sustrato por unidad de tiempo y de biomasa (g. $\left.\right|^{-1}$ DQO/ día.g.|-1 $S S)$.

$q_{\operatorname{máx}}=$ Velocidad máxima específica de eliminación de sustrato.

$\mathrm{S}_{\theta}=$ Concentración de sustrato en el reactor.

$\mathrm{K}_{\mathrm{s}}=$ Constante de Michaelis-Menten.

Previamente a la aplicación de este modelo cinético se debe comprobar que los datos experimentales se adaptan a las características de la ecuación propuesta.

Así pues para altas concentraciones de sustrato, se cumple:

$$
S_{\theta} \gg K_{s}
$$

con lo que $\mathrm{K}_{\mathrm{s}}$ puede despreciarse del denominador de la ecuación (1), quedando:

$$
q=q_{\text {máx }}
$$

ecuación que nos indica que para este intervalo de concentraciones la velocidad de eliminación de sustrato es constante $e$ independiente de la concentración de éste (cinética de orden cero).

En cambio para bajas concentraciones de materia orgánica,

$$
S_{e} \ll K_{s}
$$

con lo que $S_{\theta}$ puede eliminarse del denominador de la ecuación (1), quedando:

$$
q=\frac{q_{\text {máx }}}{k_{s}} \cdot s_{\theta}
$$

Al ser $q_{\text {máx }}$ y $K_{s}$ constantes, $q_{\text {máx }} / K_{s}=K=$ constante,

$$
q=K \cdot S_{\theta}
$$

ecuación que nos indica que para bajas concentraciones de sustrato, la velocidad de eliminación del mismo presenta una cinética de primer orden, tal como se comprueba con los datos experimentales (Tabla III).

Una vez comprobado el ajuste de los datos experimentales a las características de la -ecuación cinética 
propuesta, se determinan los valores de los parámetros cinéticos $q_{\max }$ y $K_{s}$. Para ello se ha de tener en cuenta que cuando se utiliza la DQO, como parámetro de medida de la concentración de materia orgánica de un sistema y existe una fracción de la misma no biodegradable, como es nuestro caso, la ecuación de Michaelis se puede poner de la forma:

$$
q=q_{\max } \cdot \frac{\left(S_{\theta}-S_{n}\right)}{K_{s}+\left(S_{e}-S_{n}\right)}
$$

siendo $S_{n}$ la fracción de concentración de sustrato no biodegradable (en nuestro caso, $S_{n}=0,1 \mathrm{~g} / \mathrm{l}$ ).

Tomando el recíproco de la ecuación (5) se obtiene:

$$
\frac{1}{q}=\frac{1}{q_{\text {max }}}+\frac{K_{s}}{q_{\text {max }}} \cdot \frac{1}{\left(S_{\theta}-S_{n}\right)}
$$

de forma que a partir de la representación 1/q frente a $1 /$ $\left(S_{e}-S_{n}\right)$ se debe obtener una línea recta, de ordenada en el origen $1 / q_{\max }$ y pendiente $K_{s} / q_{\text {max }}$. Esta representación gráfica conocida con el nombre de representación de Lineweaver-Burk, permite le determinación de los parámetros cinéticos $q_{\text {máx }}$ y $\mathrm{K}_{\mathrm{s}}(7)$.

En la Figura 2 aparece esta representación. Los valores de 1/q se obtienen a partir de la expresión (7):

$$
\frac{1}{q}=\frac{x}{S_{0}-\left(S_{e}-S_{n}\right)}
$$

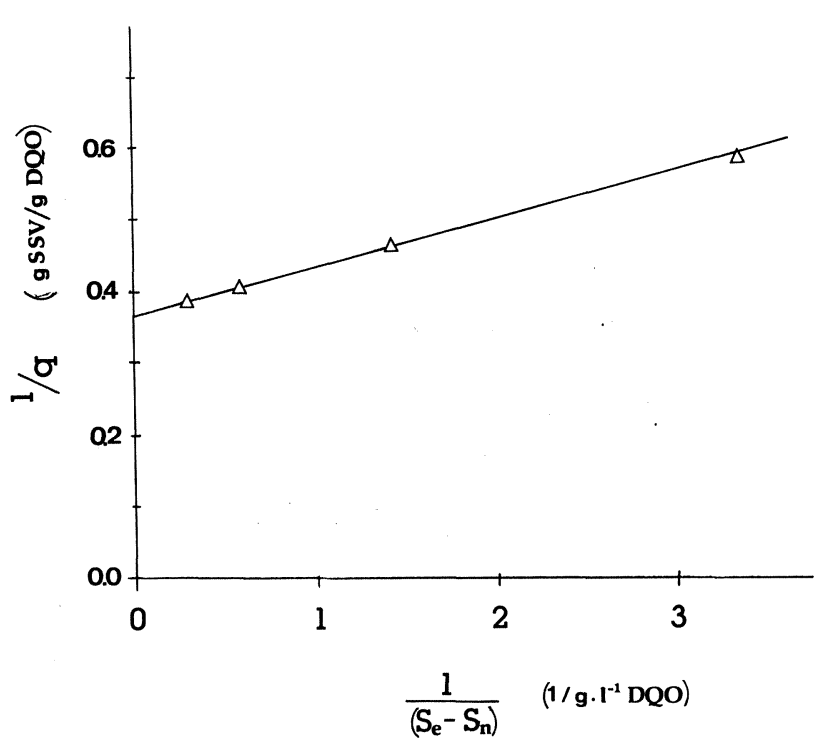

Figura 2

Representación de Lineweaver-Burk
Siendo $\mathrm{X}$, la concentración de biomasa bacteriana, medida como sólidos en suspensión volátiles (Tabla II).

$\mathrm{S}_{\mathrm{o}}$, la concentración inicial de sustrato $(4,8 \mathrm{~g} / \mathrm{l}$ de $\mathrm{DQO})$.

Las parejas de valores $1 / q, 1 /\left(S_{e}-S_{n}\right)$ se ajustan a una línea recta utilizando el método de minimos cuadrados, obteniéndose los siguientes resultados:

Ordenada en el origen $=0,36 \pm 0,02$

Pendiente $=0,07 \pm 0,01$

(Coeficiente de regresión lineal $=0,997$ )

Teniendo en cuenta la ecuación (6), se obtienen los valores de los parámetros cinéticos $q_{\max }$ y $\mathrm{K}_{\mathrm{s}}$ :

$$
\begin{aligned}
\mathrm{q}_{\text {máx }} & =2,77 \frac{\mathrm{g} / / \mathrm{DQO}}{\text { día.g/l SSV }} \\
\mathrm{K}_{\mathrm{s}} & =0,19 \mathrm{~g} / \mathrm{LQO}
\end{aligned}
$$

parámetros que representan la actividad cinética de los microorganismos responsables de este proceso aerobio.

\section{CONCLUSIONES}

1. La depuración de los efluentes resultantes del proceso de biometanización del alpechín se puede realizar vía aerobia, eliminándose más del $80 \%$ de la materia orgánica de los mismos en los tres primeros días de fermentación en régimen discontinuo.

2. Para altas concentraciones de sustrato, intervalo correspondiente a los dos primeros días de aireación, la velocidad de eliminación de materia orgánica sigue una cinética de orden cero, presentando un valor constante $(1,52 \mathrm{~g} / \mathrm{DQO} / \mathrm{di} a)$ e independiente de la concentración de aquél.

3. Para bajas concentraciones de sustrato, zona comprendida entre el tercer y sexto día de fermentación, la velocidad de eliminación de sustrato, muestra una cinética de primer orden, al presentar valores directamente proporcionales a la concentración de sustrato.

4. Por medio del modelo cinético de eliminación de sustrato de Michaelis-Menten se obtienen los parámetros cinéticos que caracterizan a este proceso aerobio.

5. Tras el proceso aerobio queda una fracción de DQO residual no biodegradable $(0,1 \mathrm{~g} / \mathrm{l})$, que es necesario eliminar mediante un tratamiento físico-químico adecuado, para obtener una depuración integral de este agua residual.

\section{AGRADECIMIENTOS}

Queremos hacer constar nuestro agradecimiento a $D^{\mathbf{a}}$ Carmen Sánchez y a $D^{a}$ Antonia Escobar por su eficaz ayuda y colaboración. 
BIBILIOGRAFIA

1. Fiestas, J.A. - Directrices actuales en la depuración de aguas residuales de carácter orgánico".- Química e Industria 30 (1984) 431-435.

2. Olthof, M. and Oleszkiewick, J. - Anaerobic treatment of industrial wastewatern.- Chemical Engineering 15 (1982) 121-126.

3. Edeline, F. -"L'épuration biologique des eaux residuaires. Théorie et technology".- Ed. Cebedoc-Liege, 1990.

4. Brock, T.D., Smith, D.W. and Madigan, M.T. --Biology of microorganisms".- Pretice-Hall International editions, London, 1984.

5. Jenkins, S.H. - Advanced treatment of wastewatern.- Ed. Oxford-Pergamon Press, 1978.

6. American Public Health Association (APHA). -"Standard Methods for the Examination of Water and Wastewatern.- 16th edition, (1985). Washington.

7. Ramalho, R.S. -mIntroduction to wastewater treatment processes".Second edition. Ed. Academic Press, New York, 1983.

(Recibido: Mayo 1991) 